\title{
Role of transfer reactions in heavy-ion collisions at the Coulomb barrier
}

\author{
Giovanni Pollarolo ${ }^{1, \mathrm{a}}$ \\ ${ }^{1}$ Dipartimento di Fisica Teorica, Università di Torino and INFN, Sez. di Torino, \\ Via Pietro Giuria 1, I-10125 Torino, Italy
}

\begin{abstract}
One and two neutron transfer reactions are discussed in the semiclassical formalism. The twoneutrons transfer cross sections are calculated in the successive approximation. Comparisons with new experimental data below the Coulomb barrier are discussed in term of transfer probabilities as a function of the distance of closest approach for Coulomb scattering.
\end{abstract}

\section{Introduction}

The degrees of freedom associated with the rearrangement of nucleons are very important in the understanding of the evolution of a heavy-ion reaction. By following the usual classification of reactions at energy close to the Coulomb barrier, we can summarize the role of transfer degrees of freedom as it follows:

- elastic scattering: they constitute the main contribution to the absorptive potential $i W(r)$,

- quasi-elastic reactions: these reactions allow the extraction of spectroscopic factors and provide information on correlations,

- deep-inelastic collision: they provide the main mechanism for the dissipation of energy and angular momentum (friction force),

- fission: they are important degrees of freedom for the dynamic of the neck rupture,

- fusion reaction: here the role of transfer is elusive but it is thought to play an important role.

In Fig. 1 is shown, for the indicated system, the excitation function for fusion processes in comparison with the inclusive cross sections for one and two neutron transfer reactions. [1]. The total reaction cross section is dominated, at low energies, by the transfer processes. These have to be driven by matrix elements that have a long tail since the distance of closest approach is, at these energies, quite outside the Coulomb barrier. It is also clear that the two-neutrons transfer process is of second order. Here we recall that the first order, one-particle transfer is proportional to the collision time while the second order is proportional at the square of the collision time.

In this talk I will restrict the discussion to transfer reactions of one- and two-nucleons at energy well below the Coulomb barrier. Even though the cross sections are quite small, advantages appear when dealing with these low energies. In this situation the two nuclei are kept apart mak-

a e-mail: nanni.to.infn.it

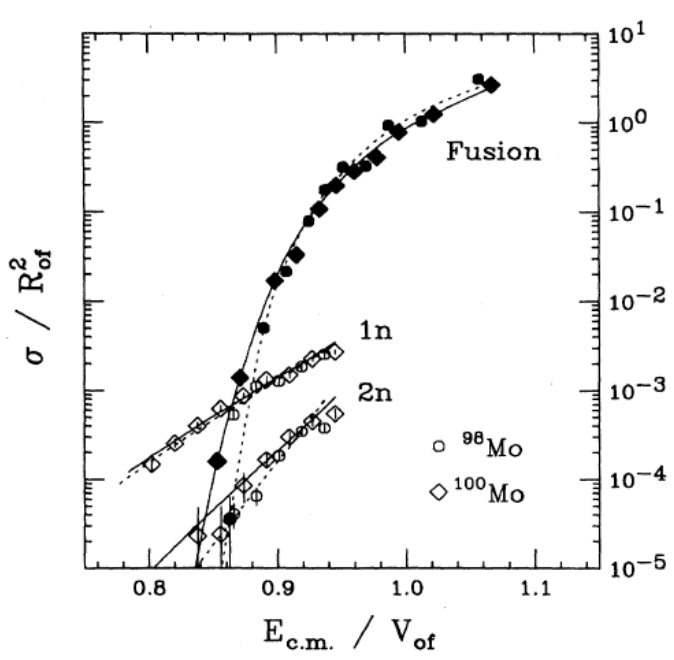

Fig. 1. Fusion excitation function in comparison with inclusive one and two neutron transfer reactions for the ${ }^{32} \mathrm{~S}+{ }^{98,100} \mathrm{Mo}$ systems.

ing negligible the formation of compound nuclei and ensure that the transfer process is a direct one. At the same time the distortion of the Coulomb elastic waves by the nuclear attraction is very small and may easily be accounted for. However, the main advantage of these low energies is that in the calculations of the transfer cross section one needs only the overlap of the tails of the intrinsic wave functions that are involved in the transition and these asymptotic behaviours are well known at least for single-particle transfer. Not only the one particle transfer reactions can be well described but at this energies one has been able to obtain a nice description of the data also for the two particle transfer channel by including contributions coming from the successive mechanism [2-4]. After a short overview of the theoretical formalism, application to new sub-barrier data [6] will be discussed. 


\section{Remarks from theory}

The transfer of neutrons and protons between two colliding ions is essentially due by the mean single-particle field (the shell model potential) of one of the two nuclei that is felt by the nucleons of the other nucleus. This process is characterized by many open channels that are all quite weak so to ensure a perturbative approach. In the semiclassical approximation, the amplitude for the exchange of one nucleon may be written as:

$$
c_{\beta \alpha}(\ell)=\frac{1}{i \hbar} \int_{-\infty}^{+\infty}<\psi_{\beta}\left|\left(V_{\alpha}-U_{\alpha}\right)\right| \psi_{\alpha}>_{\mathcal{R}} e^{i\left(E_{\beta}-E_{\alpha}\right) t / \hbar} d t
$$

where the time integral has to be performed along the classical trajectory of partial wave $\ell$. $V_{\alpha}$ represents the interaction among all the nucleons in the entrance channel mass partition and $U_{\alpha}$ is its expectation value in their groundstate configuration that is normally identified with the real part of the ion-ion optical potential. With $\mathcal{R}$ we indicated the distance between the center-of-mass of the two nuclei. In the semiclassical approximation this is defined as the average between the distances in the entrance and exit channel. The channel wave function, of energy $E_{\beta}$,

$$
\mid \psi_{\beta}(t)>=\psi_{b}\left(\xi_{b}, t\right) \psi_{B}\left(\xi_{B}, t\right) e^{-\frac{i}{\hbar} \delta_{\beta}(t)}
$$

is defined as the product of the two intrinsic wave functions times a phase factor $\delta_{\beta}(t)$ that takes into account that the two ions are moving along a classical trajectory developing in a nuclear plus Coulomb field.

By considering the stripping of a single nucleon, for even-even nuclei $\left(I_{a}=I_{A}=0\right)$, the first order amplitude for the transition of a nucleon from the single particle state $a_{1}^{\prime} \equiv\left(n_{1}^{\prime}, l_{1}^{\prime}, j_{1}^{\prime}\right)$ in the projectile to the single particle state $a_{1} \equiv\left(n_{1}, l_{1}, j_{1}\right)$ in the target may be written in the form:

$$
c_{\beta \alpha}(\ell)=-i \sum_{\lambda \mu} \frac{(-1)^{\lambda+\mu}}{\sqrt{2 \lambda+1}}<j_{1}^{\prime} m_{1}^{\prime} \lambda-\mu \mid j_{1} m_{1}>I_{\lambda \mu}^{\left(a_{1}, a_{1}^{\prime}\right)}(\ell)
$$

where $\lambda$ is the transferred angular momentum, $\mu$ its third component and $I_{\lambda \mu}^{\left(a_{1}, a_{1}^{\prime}\right)}$ is the orbital integral of the radial formfactor. This is calculated to be:

$$
\begin{aligned}
I_{\lambda \mu}^{\left(a_{1}, a_{1}^{\prime}\right)}(\ell) & =\sqrt{\frac{2 \pi a_{t r}}{\ddot{r}_{o} \hbar^{2}}} Y_{\lambda \mu}(\pi / 2,0) f_{\lambda 0}\left(\mathcal{D}_{\ell}\right) \\
& \times e^{-\frac{a_{t r}}{2 r_{0} \hbar^{2}}\left(\Delta E-Q_{o p t}-\hbar \mu \dot{\phi}(0)\right)^{2}}
\end{aligned}
$$

where with $f_{\lambda 0}$ we have indicated the radial formfactor of multipolarity $\lambda$ and with $\Delta E$ the Q-value of the transition. The transfer probability is readily calculated from the amplitude as:

$$
\begin{aligned}
P_{\beta \alpha}(\ell) & =P_{\left(a_{1}, a_{1}^{\prime}\right)}(\ell)=\sum_{m_{1}^{\prime}, m_{1}}\left|c_{\beta \alpha}(\ell)\right|^{2} \\
& =U^{2}\left(a_{1}, I_{A}\right) V^{2}\left(a_{1}^{\prime}, I_{a}\right) \sum_{\lambda \mu} \frac{2 j_{1}^{\prime}+1}{2 \lambda+1}\left|I_{\lambda \mu}^{\left(a_{1}, a_{1}^{\prime}\right)}(\ell)\right|^{2}(5)
\end{aligned}
$$

where we wrote explicitly the occupation probabilities for the single particle states $a_{1}^{\prime}$ and $a_{1}$ in projectile and target respectively.

In deriving the above expression we exploited the fact that the radial form factor, in the tail region, may be well approximated with an exponential tail:

$$
f_{\lambda 0}(\mathcal{R}) \sim e^{-\left(\mathcal{R}-R_{0}\right) / a_{t r}}
$$

and that the trajectory of relative motion may be approximated with a parabola osculating the true trajectory at the distance of closest approach $\mathcal{D}_{\ell} . \ddot{r}_{0}$ and $\dot{\phi}(0)$ are the radial acceleration and the angular velocity at the distance of closest approach. With $Q_{\text {opt }}$ we have indicated the optimum Q-value. It takes into account that the amplitude is maxima for transitions that have a good matching between the entrance and exit channels trajectories. Its expression may be found in ref. [5].

The parameter $a_{t r}$ defines the range of the transfer formfactor. Its value is related to the binding energy of the transferred nucleon in the target system if one adopt the so called prior representation while it is related to the binding energy in the projectile if one adopts the post representation. This asymmetric behaviour, it derives from the non orthogonality of the channels wave functions, is of minor importance for reactions involving nuclei in the $\beta$-stability valley but may lead to conflicting arguments when dealing with exotic beams. Here the Fermi-energies of projectile and target are very different. These considerations have also some relevance in the present talk since we chose to represent the transfer probabilities as a function of the distance of closest approach $\left(\mathcal{D}_{\ell}\right)$ (cfr. 4 and 6 ) in order to have a direct visualization of the tail of the transfer form factor. From Eq. 3 and 4 we see that the probability for a given transfer is proportional to the square of the formfactor time a factor, adiabatic cut-off function, that depends from the optimum Q-value of the reaction. It is clear that it is from this result that it is convenient to extract, from the data, the transfer probability and to plot it as a function of the distance of closest approach $\mathcal{D}_{\ell}$. However one has to keep in mind that the transfer probability extracted from inclusive cross section has a tail representing the binding energies of the transferred nucleon only if the width of the Q-value spectra does not depend strongly from the bombarding energy.

In applying the above formalism in the calculation of inclusive cross sections we cannot just add-up the probabilities of all possible transitions, the number of transitions may be very large and the sum may exceed one even if the single transitions are quite small. The inclusive transfer probability $P_{i n c}$ has to be calculated as:

$$
\begin{aligned}
P_{i n c} & =\sum_{\beta} P_{\beta \alpha} \prod_{\gamma \neq \beta}\left(1-P_{\gamma \alpha}\right) \\
& \simeq e^{-\sum_{\gamma} P_{\gamma \alpha}} \sum_{\beta} P_{\beta \alpha} \\
& =\mathrm{e}^{-\frac{2}{\hbar} \int_{-\infty}^{+\infty} W(t) d t} \sum_{\beta} P_{\alpha \beta}
\end{aligned}
$$


Our treatment of the two-particle transfer process follows very closely the one of ref. [4] so that we here simply remind some initial considerations referring to the above ref. for details. To calculate the amplitude for the transfer of two-nucleons one has to solve the well known system of semiclassical coupled equations up to the second order Born approximation. The second order Born approximation may be written in the form:

$$
c_{\beta \alpha}(\ell)=\left(c_{\beta \alpha}\right)_{(1)}+\left(c_{\beta \alpha}\right)_{\text {ort }}+\left(c_{\beta \alpha}\right)_{\text {succ }}
$$

where:

$$
\begin{aligned}
\left(c_{\beta \alpha}\right)_{\text {succ }}= & \left(\frac{1}{i \hbar}\right)^{2} \sum_{\gamma} \\
& \int_{-\infty}^{+\infty} d t<\psi_{\beta}\left|\left(V_{\gamma}-U_{\gamma}\right)\right| \psi_{\gamma}>_{\mathcal{R}} e^{i\left(E_{\beta}-E_{\gamma}\right) t / \hbar} \\
& \int_{-\infty}^{t} d t^{\prime}<\psi_{\gamma}\left|\left(V_{\alpha}-U_{\alpha}\right)\right| \psi_{\alpha}>_{\mathcal{R}} e^{i\left(E_{\gamma}-E_{\alpha}\right) t / \hbar}(9)
\end{aligned}
$$

being $(\alpha)$ the initial, $(\gamma)$ the intermediate and $(\beta)$ the final channel respectively. In Eq. 8 the first two terms arise from the non-orthogonality of the channels vectors and their contributions cancel out [2-4] so that only the last term, successive transfer, is considered for the calculation of the two particle transfer amplitude. By using the twoparticle parentage expansion of projectile $(a)$ and targetlike $(B)$ wave functions one can express the successive amplitude (cfr. Eq. 9) in term of the one-particle transfer formfactor introduced for the calculation of one-nucleon transfer reaction. Working in the m-representation one can write (cfr. Ref. [4]):

$$
\begin{gathered}
\left(c_{\beta \alpha}\right)_{\text {succ }}=\frac{1}{\hbar^{2}} \sum_{a_{1}, a_{1}^{\prime}} B^{(A)}\left(a_{1} a_{1} ; 0\right) B^{(a)}\left(a_{1}^{\prime} a_{1}^{\prime} ; 0\right) \\
2 \frac{(-1)^{j_{1}+j_{1}^{\prime}}}{\sqrt{\left(2 j_{1}+1\right)} \sqrt{\left(2 j_{1}^{\prime}+1\right)}} \sum_{m_{1} m_{1}^{\prime}}(-1)^{m_{1}+m_{1}^{\prime}} \\
\quad \int_{-\infty}^{+\infty} d t f_{m_{1} m_{1}^{\prime}}(\mathcal{R}) e^{i\left[\left(E_{\beta}-E_{\gamma}\right) t+\delta_{\beta \gamma}(t)+\hbar\left(m_{1}^{\prime}-m_{1}\right) \Phi(t)\right] / \hbar} \\
\int_{-\infty}^{t} d t f_{-m_{1}-m_{1}^{\prime}}(\mathcal{R}) e^{i\left[\left(E_{\gamma}-E_{\alpha}\right) t+\delta_{\gamma \alpha}(t)-\hbar\left(m_{1}^{\prime}-m_{1}\right) \Phi(t)\right] / \hbar}(10)
\end{gathered}
$$

The two-dimensional time integral is easily calculated in term of the one-dimensional time integral, encountered in the calculation of one-neutron transfer amplitude, by using the step-function $\theta\left(t-t^{\prime}\right)$ to complete the second time integral up to $+\infty$ and then by using:

$$
\theta\left(t-t^{\prime}\right)=\frac{1}{2}\left[1+\epsilon\left(t-t^{\prime}\right)\right]=\frac{1}{2}\left[1+\frac{i}{\pi} \mathcal{P} \int_{-\infty}^{+\infty} \frac{d q}{q} e^{i q(t-t)}\right]
$$

with the above substitution it is easy to write the amplitude for the successive transfer in the form:

$$
\begin{aligned}
\left(c_{\beta \alpha}\right)_{\text {succ }} & =\sum_{\gamma}\left[\frac{1}{2} c_{\beta \gamma}\left(\omega_{\beta \gamma}\right) c_{\gamma \alpha}\left(\omega_{\gamma \alpha}\right)\right. \\
& \left.\times \frac{i}{2 \pi} \mathcal{P} \int_{-\infty}^{+\infty} \frac{d q}{q} c_{\beta \gamma}\left(\omega_{\beta \gamma}-q\right) c_{\gamma \alpha}\left(\omega_{\gamma \alpha}+q\right)\right](12)
\end{aligned}
$$

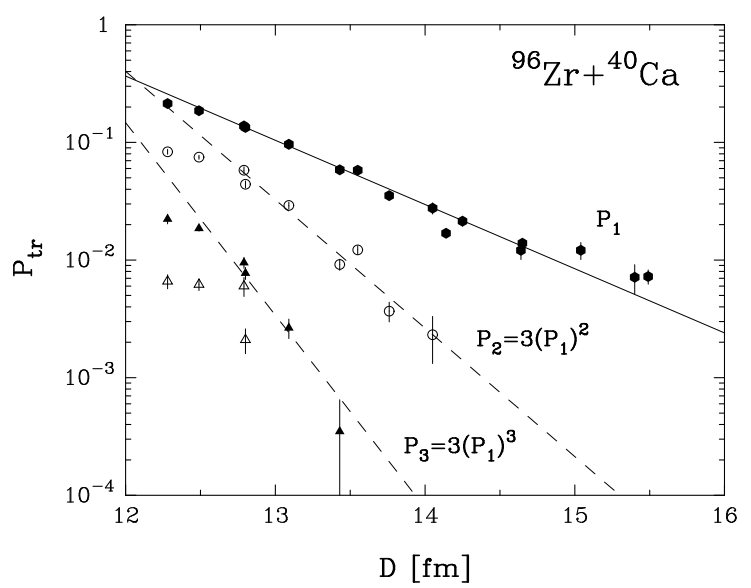

Fig. 2. Transfer probabilities for neutron-stripping channels as a function of the distance of closest approach.

The first term in this expression, real, corresponds to transitions on the energy-shell since the energy entering in the first-order amplitudes are the one corresponding to the physical transitions. The second term, purely imaginary, corresponds to transitions off the energy-shell since the energy are here shifted by an amount $q$. The real part of the successive amplitude provides the dominant contribution for transition where the pair of nucleons has a small correlation energy. The imaginary part, instead, provides the dominant contribution when the correlation energy of the pair is large. Also for two particle transfer the probability is obtained by squaring the amplitude and multiplying the result for the probability to remain in the elastic channels as in eq. 7.

\section{Data analysis}

The semiclassical formalism outlined above will be applied to the analysis of the ${ }^{96} \mathrm{Zr}+{ }^{40} \mathrm{Ca}$ reactions. The experiment was performed in inverse kinematic with a ${ }^{96} \mathrm{Zr}$ beam delivered from the XTU-Tandem + ALPI superconducting booster of LNL. It was possible to extract excitation functions up to the transfer of 4 neutrons in the energy range between 330 t0 $255 \mathrm{MeV}$ of bombarding energies (for detail we refer to the talk [6] in this conference). From the experimental cross sections the transfer probabilities $P_{t r}$ are extracted from the formula:

$$
P_{t r}=\frac{d \sigma_{t r}}{d \sigma_{\text {Ruth }}}
$$

and plotted as a function of the distance of closest approach $D$ for a Coulomb trajectory,

$$
D=\frac{Z_{a} Z_{A} e^{2}}{2 E_{c . m .}}\left(1+\frac{1}{\sin \left(\theta_{c . m .} / 2\right)}\right)
$$

for the $+1 n,+2 n$ and $+3 n$ transfer channels these are shown in Fig. 2.

The transfer probabilities are very well described with an exponential function with a decay length that gets smaller 


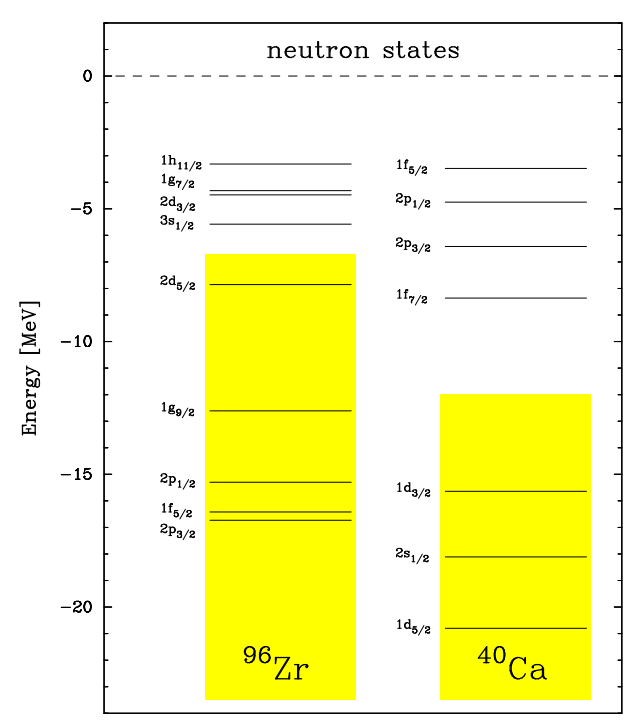

Fig. 3. Neutron single particle levels in ${ }^{96} \mathrm{Zr}$ and ${ }^{40} \mathrm{Ca}$.

as the number of transferred neutrons increases. This behaviour of the transfer probabilities suggests a simple phenomenological interpretation of the data. The full line represents a fit to the transfer probability for the $+1 n$ channel. By using a simple transfer model based on the exchange of independent particles that predicts for the two particle channel a probability proportional to the square of the single particle probability, one can obtain a nice description for the $+2 n$ and $+3 n$ channels with the equations $P_{2 n}=3\left(P_{1 n}\right)^{2}$ and $P_{3 n}=3\left(P_{1 n}\right)^{3}$. These are shown with dash lines in the same figure. The factor 3 appearing in the previous expressions is usually referred as enhancement factor and should represent a measure of the collectivity of the transferred pair.

The comparison between the theory and the experiment starts by calculating the inclusive one-neutron stripping process. Since projectile and target may be both considered closed shell nuclei we will describe the spectra of ${ }^{95} \mathrm{Zr}$ as pure hole states while the one of ${ }^{41} \mathrm{Ca}$ as pure particle states. In this approximation the inclusive probability for one-neutron stripping may be calculated (from Eq. 7) by summing over all single particle transitions that can be constructed from the single particle levels shown in Fig. 3. The result of such a calculation is shown, with a full line in Fig. 4 in comparison with the experimental data [6].

The data are quite well described both in magnitude and slope. The reproduction of the slope is not a surprise since the one neutron transfer formfactors are constructed by using the experimental single particle energies. The spectroscopic factors have been all set to one, in fact dealing with inclusive cross section we can neglect the fragmentation of the single-particle strength on several states and suppose that the full strength is concentrate on a single transition.

Before the discussion on two-particles transfer, it is instructive to have a look at the Total Kinetic Energy Loss (TKEL) distributions that are shown in Fig. 5 for the indicated bombarding energies. The quasi-elastic channel (first

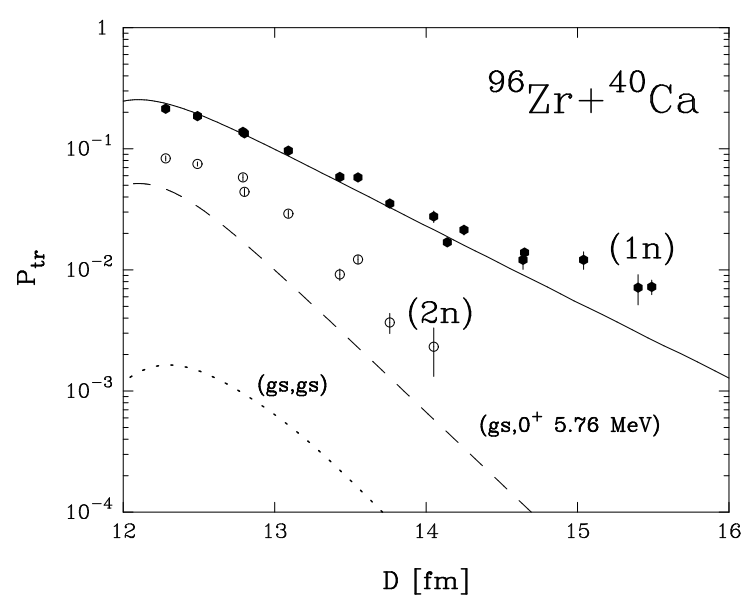

Fig. 4. Theoretical transfer probabilities for one and two particles transfer in comparison with the experimental data. The full line represents the inclusive transfer probability for one-neutron transfer, the dotted line represents the ground-ground states transition for the two-neutrons transfer channel and the dash line the transition to the first $0^{+}$excited state at $5.76 \mathrm{MeV}$ for the same channel.

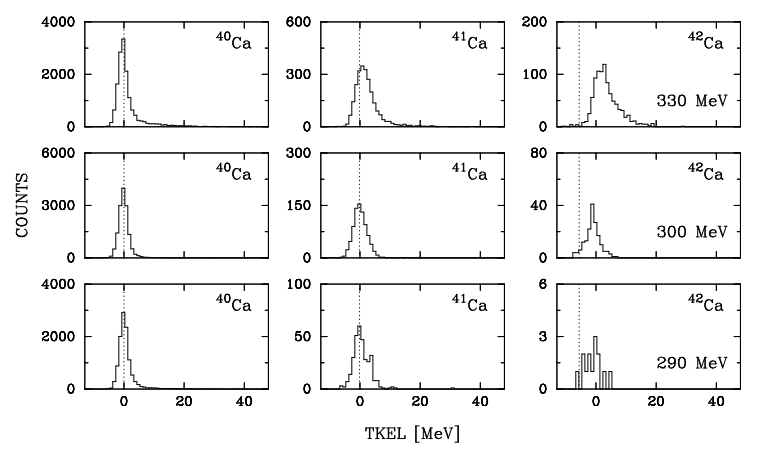

Fig. 5. Experimental TKEL distributions for the elastic(+inelastic) and $+1 n$ and $+2 n$ transfer channels obtained in the reaction ${ }^{96} \mathrm{Zr}+{ }^{40} \mathrm{Ca}$ at the indicated bombarding energies. The vertical dotted lines represent the ground state Q-values.

column) has a width of $\sim 3 \mathrm{MeV}$ that is compatible with the overall resolution of PRISMA and of the beam energy indetermination. The spectra for the $+1 n$ channel (middle column) have a maximum that slightly moves toward higher energy losses with the increase of the bombarding energy, in accordance with the energy dependence of the optimum Q-value that, for neutrons, is very closed to zero. The width of these spectra is constant below the Coulomb barrier and grows above it reflecting the opening of reaction channels other then the ground states transition. The spectra of the $+2 n$, last column, display a similar behaviour but, in this case, the reaction mechanism leaves unpopulated the ground state transition where most the pairing strength should be concentrated. The vertical dotted lines, in the frames, indicate the ground states Q-values, they are at $+0.14 \mathrm{MeV}$ for the $+1 n$ channel and at $+5.6 \mathrm{MeV}$ for the $+2 n$ channel. 
Table 1. Wave functions for the $0^{+}$states in ${ }^{42} \mathrm{Ca}$ deriving from the Tamm-Dancoff approximation

\begin{tabular}{lrrrr}
\hline $\mathrm{E}\left(0^{+}\right)$ & $f_{7 / 2}$ & $p_{3 / 2}$ & $p_{1 / 2}$ & $f_{5 / 2}$ \\
\hline 0.00 & 0.93 & 0.22 & 0.19 & 0.21 \\
5.76 & 0.29 & -0.93 & -0.16 & 0.14 \\
9.10 & 0.20 & 0.27 & -0.86 & -0.38 \\
\hline
\end{tabular}

For the two-particle transfer channel it is interesting to see if our formalism is able to predict the very small population of the ground states and if it concentrates the transition strength at an energy loss of the order of $6 \mathrm{MeV}$. To calculate the ground states transition we need a microscopic description of ${ }^{94} \mathrm{Zr}$ and ${ }^{42} \mathrm{Ca}$. For the ground state of ${ }^{94} \mathrm{Zr}$ we perform a simple BCS calculation with a state independent pairing interaction with a strength $\mathrm{G}=0.218$ $\mathrm{MeV}$. This interaction provides a pairing gap $\Delta=0.746$ $\mathrm{MeV}$ and a Fermi energy at $7.6523 \mathrm{MeV}$. The BCS calculation has been performed by using the single particle states shown in Fig. 3. For the ${ }^{42} \mathrm{Ca}$ we have performed a simple shell model calculation by using the shell model space provided by the set of single particle levels outside the core $\left({ }^{40} \mathrm{Ca}\right)$ that are shown in the same figure. The residual interaction that binds the two neutrons is a simplified delta interaction (see ref. [7]). Diagonalizing this interaction we obtain for the $0^{+}$states the one shown in Table 1 . The strength of the interaction is fixed so to give the right separation energy between ${ }^{40} \mathrm{Ca}$ and ${ }^{42} \mathrm{Ca}$. The model predicts a $0^{+}$state at $5.76 \mathrm{Mev}$ that we would like to identify with the state at $5.87 \mathrm{MeV}$ that is strongly populated in $(t, p)$ reaction [8]. Since in our model space there is no room for the excitation of the core, the model is not able to describe the $0^{+}$state at $1.837 \mathrm{MeV}$. This state should be little populated by a two-particle transfer mechanism. In fact it requires a higher order process like transfer followed by an inelastic excitation or vice versa.

In Table 1 are shown the energies and the wave functions obtained with the above calculation. It is interesting to notice that the predicted ground state is dominated by the two neutrons in the $f_{7 / 2}$ orbital while the excited states at $5.76 \mathrm{MeV}$ has a wave function dominated by the two neutrons in the $p_{3 / 2}$ orbital. This is very important since the $f_{7 / 2}$ orbital has a wave function with a much smaller tails (then the $p_{3 / 2}$ ) because of the larger centrifugal barrier. This simple fact reflects itself in the formfactor that is much larger for the transition that leaves the neutrons in the $p_{3 / 2}$. Using the above wave functions the groundground state transition is predicted to have a probability represented by the dotted line in Fig. 4. It is clear, its contribution is negligible.

Now evaluating the transition to the $0^{+}$state at 5.76 $\mathrm{MeV}$ (the ${ }^{94} \mathrm{Zr}$ is left in its ground state) we obtain the result shown with a dash line in the same Fig. 4. This cross section is a factor 10 larger then the ground state transition but it is still underestimating the experimental data by almost a factor 3 . The cross section to the $0^{+}$state at 9.10 $\mathrm{MeV}$ is very small and it is not shown in the figure.

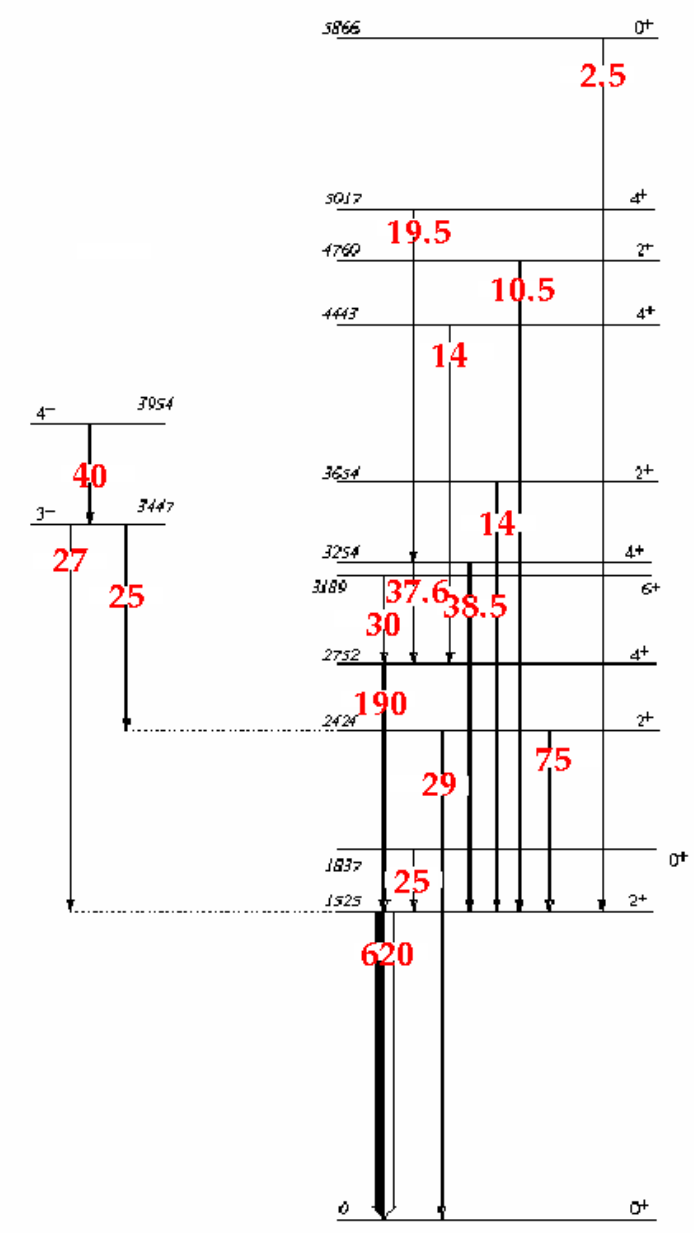

$42 \mathrm{Ca}$

Fig. 6. ${ }^{42} \mathrm{Ca}$ spectra as reconstructed from the $\gamma$ detected in coincidence with the projectile like fragment in the collision of ${ }^{40} \mathrm{Ca}$ on ${ }^{90} \mathrm{Zr}$ at $350 \mathrm{MeV}$ of bombarding energy. The numbers labelling the different transitions represent the extracted strength.

The fact that the transition to a single state $\left(0^{+}\right)$underestimates the measured inclusive cross section should not come as a surprise. From a previous measurement of the same reaction, where the $\gamma$-rays were measured in coincidence with the reaction fragments (we used the PRISMA + CLARA apparatus), we know that the $2 n$-transfer reactions are much richer than the one we have depicted. In Fig. 6 is shown the reconstructed spectra, from the detected $\gamma$ 's, for ${ }^{42} \mathrm{Ca}$ nucleus together with the estimated strength of the different transitions. One can see immediately that not only $0+$ states are populated in the reaction but also states with relatively high angular momentum and states with abnormal parity (we recall that $I_{a}=0$ and $I_{A}=0$ ). It is important to stress the population of the negative parity states since this indicates that in the $2 n$-transfer reaction the recoil term plays an important role. We cannot hope to be able to reconstruct from the $\gamma$-spectra the true population of the states via the transfer mechanism but, from a careful analysis of the strength distribution, one sees that 
the population of negative parity states and of states with large angular momentum amounts to almost $80 \%$ of the total strength. The total strength may be estimated by adding the strengths of the last $2+$ states in both nuclei. We remind that the abnormal parity states and the states with angular momentum larger then 0 cannot be calculated with our formalism.

\section{Conclusions}

The excitation functions for multinucleon transfer channels measured in a broad energy range below the Coulomb barrier have been analyzed in a semiclassical formalism that calculates the two particle transfer in the successive approximation. The formalism provides a quite good description of the transfer probability for one-neutron transfer. The transfer of $I=0^{+}$pair is shown to populate very little the ground state transition but it accounts for almost one third of the total strength if one considers the transition to the $0^{+}$at $5.76 \mathrm{MeV}$ in ${ }^{42} \mathrm{Ca}$ that has a wave function dominated by the pair on neutrons in the $p_{3 / 2}$ orbital.

\section{References}

1. R.B. Roberts et.al. PRC 47 (1993) 1831

2. B. F. Bayman and J. Chen, Phys. Rev. C 26, 1509 (1982).

3. E. Maglione, G. Pollarolo, A. Vitturi, R. A. Broglia and A. Winther, Phys. Lett. B 162, 59 (1985).

4. J.H.Sorensen, A.Winther Nucl.Phys. A550, 306 (1992)

5. R.A. Broglia and A. Winther, Heavy Ion Reactions (Addison-Wesley Pub. Co., Redwood City CA, 1991).

6. L. Corradi, this proceedings.

7. R.D. Lawson, Theory of the Nuclear Shell Model (Clarendon Press, Oxford, 1980).

8. D. C. Williams, J. D. Knight and W. T. Leland Phys. Rev. 164, 1419 (1967). 